Writing in Multiple Disciplines, Three Local Contexts, and One Global Conversation: An Online Collaboration Among Swedish and American Students - Proceedings for Computers and Writing Conference 2004

\title{
Cross-Cultural Collaborations
}

Magnus Gustafsson, Department of Language and Communication, Chalmers Lindholmen University College, Göteborg, Sweden

Donna Reiss, Department of Languages and Speech, Tidewater Community College-Virginia, USA

Art Young, Department of English, Clemson University, Clemson, South Carolina, USA

\begin{abstract}
During Fall Semester 2003, three teacher-researchers from Sweden (Chalmers Lindholmen University) and the United States (Clemson University and Tidewater Community College) coordinated a writing project in which asynchronous communication technology enabled students from three different academic contexts to communicate online with a common emphasis on effective communication within and beyond their disciplines. Students focused on their own literacy practices for a diverse, responsive audience. They articulated their understanding of the tools with which they communicate and reflected on themselves and others as communicators in contexts broadened across international borders by information technology. The many metacognitive responses in students' online postings reveal personal, social, rhetorical, and educational dimensions to their online writing that can inform future practices for writing and digital media.
\end{abstract}

\section{Proceedings}

During Fall Semester 2003, the three of us teacher-researchers from Sweden (Chalmers Lindholmen University) and the United States (Clemson University and Tidewater Community College) coordinated a writing project in which asynchronous communication technology enabled students from three different academic contexts to communicate online with a common emphasis on effective communication within and beyond their disciplines. Students focused on their own literacy practices for a diverse, responsive audience. They articulated their understanding of the tools with which they communicate and reflected on themselves and others as communicators in contexts broadened across international borders by information technology.

Approximately 240 web postings of about 350 words each by 5 groups of students in semithreaded discussion forums have been compiled. Every group included students from each of the three academic and cultural contexts. Discussion was initiated by open questions related to expectations on writing, writing with computers, and writing challenges; however, the discussion was subsequently student-centered and -controlled. We designed four discussion board prompts to build a sense of community among group members as well as to generate students' reflections on themselves as communicators first and then as communicators using technology and as communicators in an international conversation aided by technology. Our goal for students in 
these quite-different writing classes was to encourage self-examination and heightened awareness of the rhetorical situations in which they would find themselves as writers in the academy and workplace. We subsequently are analysing the data for recurring themes related to the act of writing and to communication technology and to the connection among such themes.

Our design for the assignment was informed by the theoretical foundation established by James Britton (1975, 1993). We hoped the international conversation conveyed with a discussion board would demonstrate ways information technology can support both "expressive" discourse as a writing-to-learn tool and also "transactional" discourse as a writing-to-communicate tool across cultures and educational levels. Our collaborative analysis and formal analyses by three Clemson University graduate students as well as informal analyses by selected students from all three contexts are represented in our presentation.

In addition to the conclusions about writing and about communication technology shared by many of the students, tensions in the students' online postings are emblematic of a recent statement by Bjork et al.: "Before we know it, the genres we teach today, the essay and the traditional academic research paper may be supplemented with new genres such as hypertext, calling for new ideas on how to teach and how to organize writing instruction” (15). The many metacognitive responses in students'online postings reveal personal, social, rhetorical, and educational dimensions to their online writing that can inform future practices for writing and digital media.

The Interface

We used Claroline, the course management software at Chalmers Lindholmen University, rather than the systems available at our American colleges, Blackboard, WebCT, or Clemson University's Collaborative Learning Environment. Anton Vialle, Claroline administrator, and Magnus Gustafsson made it easy for us to assign our students to a virtual class and to place them in discussion groups. Even though it was possible to construct such a class in our U.S. systems, administration and support would have been more complex and time-consuming. Because U.S. students were already accustomed to the basic operation of discussion boards, adjusting to Claroline was not a problem for most. Interestingly, despite the fact that Claroline is available in a number of languages and we used an English language version, some Swedish appears at the opening screen to remind us we are the guests of a Swedish university. 


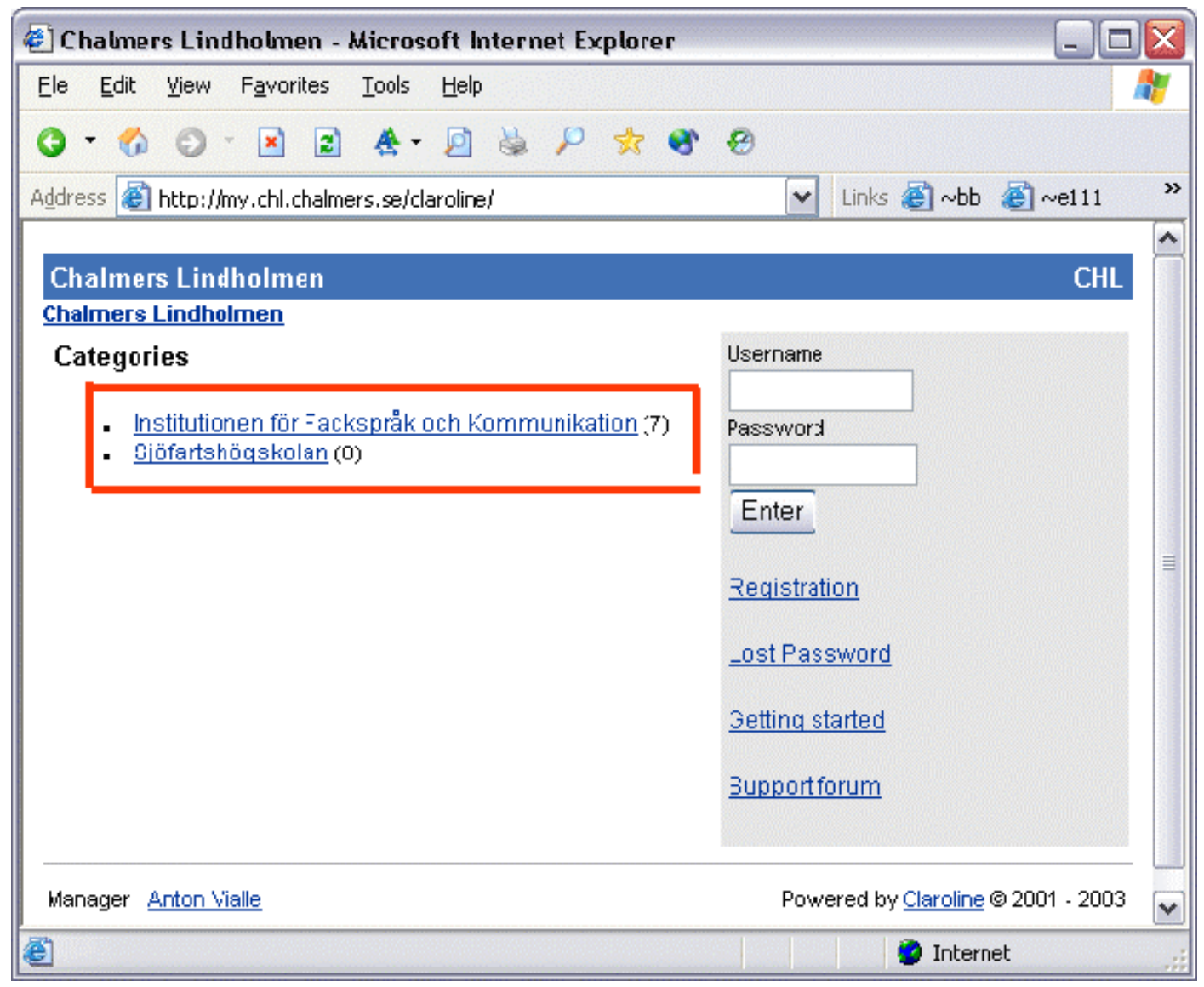

Three Contexts

Chalmers University of Technology, Göteborg, Sweden, is a research intensive university where most graduate classes are conducted in English. Doctoral students across all engineering disciplines are offered a class in academic writing to prepare for writing research articles and dissertations in their fields. As participants in global research, these graduate students must learn to communicate effectively in their fields and to communicate effectively in English which is predictably not their first language. This seminar meets once a week, and the writing is geared towards their research articles and the genre awareness needed for their future careers as writers in a research-intensive environment. These hard science researchers, despite various personal backgrounds and narratives, consider writing first and foremost as a tool to establish themselves as researchers and publish their results.

Clemson University is a land-grant university in South Carolina, and the students in our exchange are senior English education majors or Master's-level graduate students enrolled in a required class, Composition for Teachers. They are studying theories of composing and best practices in the teaching of writing, including writing across the curriculum, and are writing themselves in a variety of genres: journals, letters, reports, e-mails, discussion boards, and critical essays. The international online exchange is a key part of their portfolio and accompanying reflective analysis. Many of these students, who plan to teach writing and literature in American high schools, express apprehension about using computers in teaching. 
Tidewater Community College in Southeastern Virginia is a large four-campus commuter college, and the students in our online exchange, unlike the Chalmers and Clemson students, are not studying writing at all but are participating in a writing-intensive learning community. Their totally online humanities elective focuses on technology and society with an emphasis on technology and the arts. Communication technologies are integral to this class, which "meets" several times a week on a discussion board where students write 500 or more words of informal discussion on class topics. At semester's end, they develop a digital portfolio of their formal and informal compositions. For these students who never meet their professor or each other in person, writing has been their instructional medium, but prior to the exchange, they had not attended directly to the act of writing or its implications.

\section{Five Groups}

Past experience with online communication projects suggested that we should assign online groups larger than in-person class groups because participants who have time, work, or family conflicts may skip a session or two. Our three classes were different sizes, so we established groups of nine or ten with representatives from each college in each group: from Chalmers, two or three students; from Clemson, four or five students; and from Tidewater, three or four students. Fortunately, most students were able and willing to contribute actively to most of the assigned letters. For instance, one Clemson student vacationed in France over Fall Break and wrote to her group from a cybercafe in Paris, and one TCC student participated in the discussion from her home in Kapolei, Hawaii, where she had moved from Virginia during the academic year.

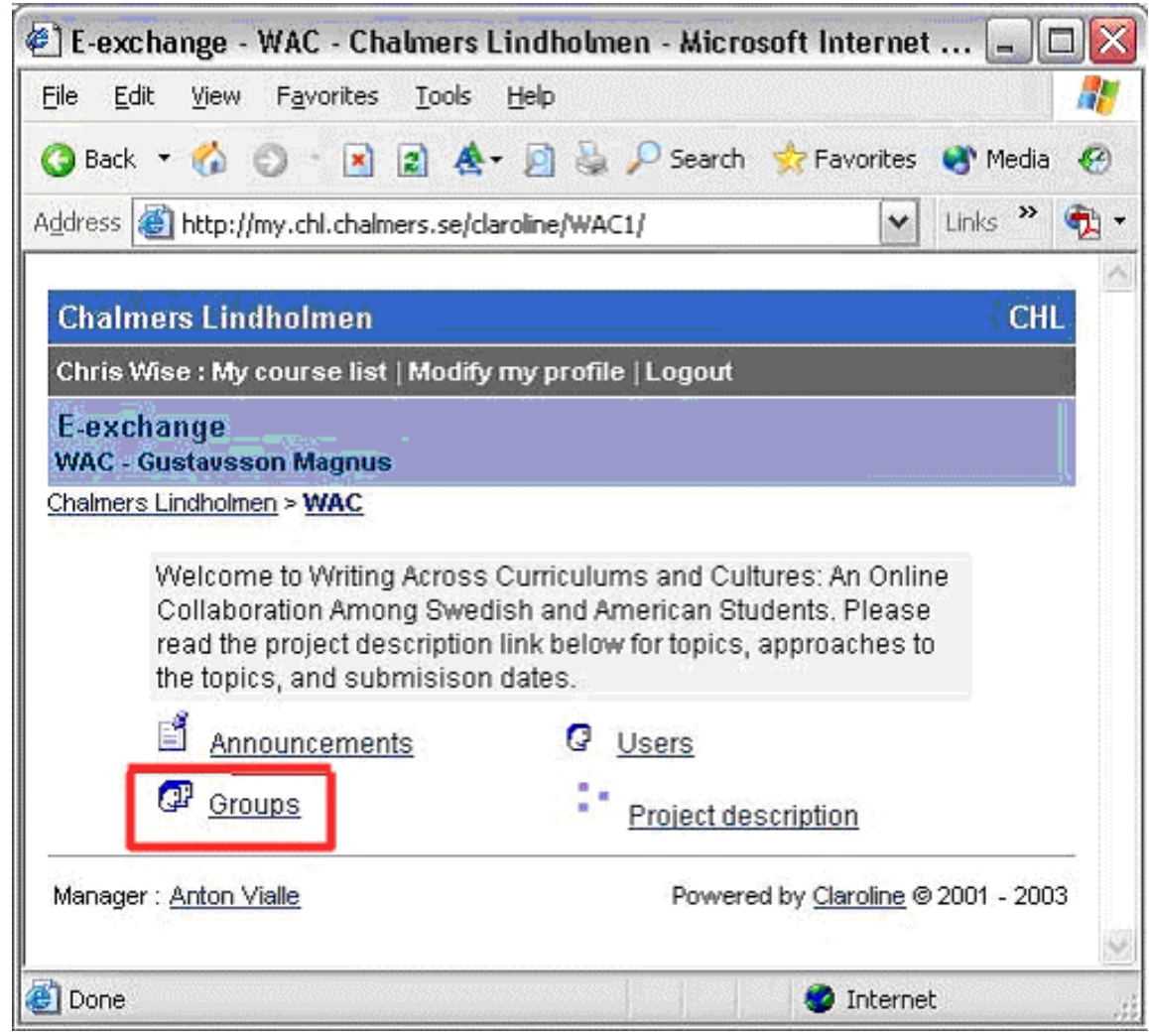




\section{Four Prompts}

The prompts for this cross-cultural exchange were posted on the Web so that students could refer to them at their own convenience no matter their location or time zone:

http://onlinelearning.tcc.edu/faculty/tcreisd/projects/cmnc03fa/ . We hoped that the expectations would be clear and explicit enough to guide the focus of the discussion but still allow some room for students not to feel overly constrained.

We arranged the four prompts in a sequence that suggests a general movement from personal to public and from narrative to analytical, thus encouraging the rhetorical development of "expressive” to "transactional” suggested by Britton and his colleagues (1975). Our conscious decision to personalize the conversation by asking students to refer to each other by name and to frame the interactions in a familiar genre, letters, may have contributed to the cohesiveness and comfort of the groups. Although we did not provide specific guidance on diction, style, usage, and tone, students demonstrated an awareness of these rhetorical features in their posts, and our analysis identifies some of the ways that students' styles were affected by the evolving discussions as well as their increasing identification with members of their groups.

\section{Letter 1}

Letter 1, approximately 350 words, addressed to everybody in the group and submitted by November 5, 11:59 p.m. Describe an experience that illustrates your perception of yourself as a writer, perhaps a situation in which writing was challenging or exciting or otherwise important. As you reflect on that experience, what were your expectations about yourself as a writer and what was the outcome of the experience? Include within your letter one or two sentences that introduce you to the group, for example, your name, which class you are taking, and your academic interest or emphasis.

\section{Letter 2}

Letter 2, approximately 350 words, addressed to everybody in the group and submitted by November 9, 11:59 p.m. Read the letters from all the members of your group and write a personal response about some of the issues raised there. In your Letter 2, addressed to your entire group, refer specifically to at least two members of the group by name, identifying the elements of their letters that were of particular interest to you. Comment on ways in which their experiences are similar to and/or different from your own. Before you compose your Letter 2, first read any second letters already posted by groupmates. Try to cite two groupmates whose Letter 1 submissions have not already been cited by others when you compose your own Letter 2.

\section{Letter 3}

Letter 3, approximately 350 words, addressed to everybody in the group and submitted by November 11, 11:59 p.m. Read the second letters from all the members of your group. Based on 
the letters and your own further thinking, speculate on the impact that communication technology is having and will have on the kinds of writing experiences you and your group have had in the past and may have in the future. Think in particular of communication in local, national, and global contexts. Incorporate into your letter: at least one reference to a groupmate's Letter 2 and at least one reference to a Website that addresses one or more of the issues raised by you and/or others. Copy-paste the entire Web address including http:// and leave a space before and after so that the discussion board will post it as an active link for your readers to follow.

\section{Letter 4}

Letter 4: Analysis of and Reflection on the conversation, approximately 350 words addressed to everybody in the group and submitted by November 16, 11:59 p.m. Read the third letters from members of your group. Then reflect on the exchange of three letters among members of your group and speculate on the key issues. Suggested topics for your Analysis and Reflection include: What are some similarities and differences among the writers in different locations and as an audience for writing? What interested you? What surprised you? What troubled you? What are some benefits and drawbacks of a letter exchange that uses a Web discussion board? What have you learned about your own expectations about writing for a distant and diverse audience?

\section{Assessment}

Students were assessed and credited differently in the three classes. Whether the assignment received little credit or considerable credit, students responded as if the conversation itself and the topics were important to them.

Magnus Gustafsson's Ph.D. class in academic writing at Chalmers Lindholmen University is a pass/fail course, and participation in our letter exchange was compulsory. He did not grade the individual letters.

Students in Donna Reiss's humanities class at Tidewater Community College also were required to participate, and their four letters were graded individually and credited collectively as a class project, 20 percent of their grade. So long as students wrote in response to the prompt directions, they received maximum credit for each submission.

Clemson University students were required to participate as well. Their discussion board letters were part of their final portfolio of written work for the course. They were not graded separately, but these discussion board entries counted for approximately 10 percent of their final grade.

Analysis

Preliminary findings suggest the following:

Tension between anxiety and rewards of writing form a background to the fear of failure that many students expressed, a fear that may be growing because of the ubiquity of the written word through the World Wide Web. For example, the Swedish students tended to mention the writing process explicitly, and the U.S. students more often commented on writing as generative. These 
two strands came together in comments related to the element of writing under pressure to meet deadlines and audience expectations. With few exceptions, only the U.S. students frequently mention the creative aspects of writing, and they often make references to writing for personal and educational purposes, while the Swedish students frequently focus on business/work contexts or academic/professional contexts, such as composing conference presentations or writing research articles for publication, as required by their doctoral programs. These two dimensions of writing might also reflect not only the background and experience of the respective groups but also the actual expectations the students had/have on writing. The following examples from Sweden and the U.S. illustrate these approaches.

I began putting the concept of the poster together....Also I tried to write on a level so that a wider group of scientists could understand our research and give us input from their point of view. When the basic layout and text where finished I send a copy to each member of our group for comments. My view of what I had written was that it was almost nothing on the poster that they could point out as unclear or unimportant for the understanding of our research. The response that I got from two of the other three members were full of suggestions to make the text more clear-cut and easy to follow. I was shocked! After three more rounds in the ring with my cowritters we finished the poster. Still licking my wounds I realised that I had some more to learn about academic writing. (Mikael, Chalmers, Letter 1)

Writing is big part of my daily activities and has been for sometime now. I really enjoy the process and I find it is a good release when you just need to get some emotions out. When most people that know me hear about my feelings towards writing, they don't see it. They see me as an emotionless being, which I must say I know why. I am quiet and usually very calm....My writing usually takes forms of quick poems or journal entries that I keep in a notebook and out of others hands. I love to write but I hate writing for other people. Writing is my release, and I don't like to share my feelings. As long as they are written down somewhere, that's good enough for me....As a writer, one day I hope to be able to write without fear that someone will tear it apart. (Charlie, Clemson, Letter 1)

Difficulties with writing included diction and genre knowledge. The forum itself highlighted the difficulty of adjusting stylistic levels to audience expectations. Swedish students opted for more formal, professional language, and U.S. students opted for more informal, conversational language. Yet, Swedish students wrote very specific and engaging personal narratives while American students often provided brief narratives and then a reflection on their thoughts and feelings about the experience. While proficiency level to some extent affects levels of formality, suggesting that Swedish students felt more comfortable with the more formal language they encounter in their research, there was a general movement in both directions: Swedish students experimented with more informal letters and American students attempted more succinct and specific posts.

For example, in Letter 1 Lindsey (Clemson) uses the salutation "Dear Groupies," and then in Letter 2 Rebecca (TCC) begins "Hello Groupies (I believe we should adopt this as our group call sign)," and then a few hours later, Gabriel (Chalmers) begins "Hello Groupies” (I am supporting Rebecca's motion).” Gabriel's first sentence then follows: "Guys, I am really enjoying your 
letters!” Gabriel followed with a sentiment many students in the exchange acknowledged over the course of the project: "It has not been easy to follow such amount of different ideas, comments and experiences regarding writing plays, creative writing, maintenance procedures, résumés, memos, formal and informal letters, and other kinds of impressive writing. I am really learning from you guys." Thus, the use of the conversational and slang "guys" to refer to both men and women and the expressive exclamation mark confirm a growing use and acceptance of conversational English within the context of this discussion board.

Here are further examples of the metacognitive discussion about such language use. Students have reflected on the writing experiences of members of their groups as well as their own. In their posts, they address the implications of audience, purpose, and voice without using those terms.

All of you have reminded me that writing is a part of everyday life (I've been out of the loop for a few years with my kids). For those of you in the business world, I am sure you understood Gabriel's reference to memo writing. That is something they should teach in college. I know I have ruffled my share of feathers around the office. From sociology I learned about "report talk" vs. "rapport talk". Report talk is the exchange of facts and pertinent information where rapport talk is a way of relating information on a more personal level. If we all were able to have rapport with others, then perhaps our reports would be more interesting. I really enjoy communicating with all of you. I hope you enjoy academic, as well as personal, success. (Rebecca, TCC, Letter 2)

Hello Groupies,... For me it is clear that the ability to write in a language other than your native language is somewhat limited. In formal writing, like writing technical and academic papers, it may be overcome or at least improved by education and practice. But in informal communication this limitation becomes more evident, especially when slang words and phrases beyond the "school English" are frequently used. (Martin, Chalmers, Letter 4).

When I first approached this project, I thought I was going to have to write these formal letters with perfect grammar and very little or no slang usage so that I would e understood. But then I remembered that this is a learning experience for you too and I took to writing like I normally would here and hoped that anything that wasn't understood would be cleared up later by me answering a question or two. I had no questions, which leads me too believe that you have a better grasp of English than I had anticipated, awesome, that made it easier on me to be a little more open. (Charlie, Clemson, Letter 4).

There is a way that some popular magazines take on highly technical, scientific topics and opens them up to a much wider audience. One useful purpose that this serves is making science and technology approachable. Too often, professional writing seems designed to keep the information "within the fold." (TJ, Clemson, Letter 2) 
Finally let me try to express my fillings regarding writing to people of different cultures and backgrounds. It has not been easy because after Letter 1 I realized that you guys know much more about writing than I do. I am a scientist and I have been writing for long, but technical papers, nothing like poems, plays or novels. What we have been doing here is completely different, because is related to emotions, personal experiences and opinions. It scared me but at the same time it taught me a lot. Cheers, (Gabriel, Chalmers, Letter 4)

Exploring the impact of information technology on writing, on communication, and on international expression from the perspective of students with a wide range of educational and career goals was one of the primary purposes for this exchange. Although all our communications were conducted in English, Swedish students were writing with a second (and in some cases third) language at a level so stylistically sophisticated that many students from the United States were impressed — and said so. The immediacy of the Internet exchange highlighted differences between Swedish and American students' writing in formality and in concern for correctness and precision in diction and syntax. At the same time, the lack of visual information and names that were not automatically identified with gender meant some familiar cues for responding to other people were absent.

One of the things I noticed during the letter exchange was that sometimes it was hard to differentiate the age and gender of fellow group members. I noticed that people referred to Xing and Erin as both he and she. Sometimes cultural differences make it difficult to distinguish whether names are feminine or masculine. This is a simple example of the confusion that internet communication can cause. Without knowing the tone of someone's voice, it is harder to sense sarcasm or humor. It also becomes difficult to know exactly who you are talking to. (Lindsey, Clemson, Letter 4).

As they considered the ways the Internet has affected their writing, some students from all three colleges expressed a preference for handwritten paper letters for personal communication while others highly valued instant messaging and email for connecting to close friends or keeping in touch with distant colleagues and family members. One Chalmers student wrote that he had met a woman at a conference in Australia, corresponded with her by email for a time, and now she was living with him in Sweden. TCC students associated with the military had loved ones in Iraq during this project, and the immediacy of email was a lifeline for them.

Erin mentioned in her letter 2 that she now write just about as many letters as she does emails. However, I use emails and instant messages all the time, because I feel they are just like letters but the delivery time goes from days and weeks to seconds. Perhaps email can be a good way to encourage informal writing, mentioned in Jennifer's letter, since it is more towards the individual than any other form of communication....Without the understanding of effective writing skill, those technologies are less useful....Talk to you next time! (Xing, Chalmers, Letter 3) 
But I still believe that computers are eventually going to make us lazy. I can remember how exciting it was t get actual letters in boot camp. That was the best part of the whole 9 weeks, that every Sunday we got our mail. I still get letters though because most people in Kentucky that I know don't have computers so I still get the jiggles and the smiles when I go to the mailbox.... (Lesley, TCC, Letter 4)

Another important factor was availability of information. Students recommended to each other Websites they had located about language and writing and technology. A TCC student emphasized the value of the Web for providing access to multiple perspectives for understanding complex issues such as the contrasting ways the media in various countries represented the United States' presence in Iraq, notably USAToday and al-Jazeera. The following examples illustrate some of these issues.

\section{Hello Groupies,}

Instantaneous communication across physical borders and political ideologies allows global access to varying perspectives throughout our world....American publications almost always refer to anti-American Iraqis as insurgents, whereas al-Jazeera describes them exclusively as resistance fighters. These two terms have opposite connotations describing the exact same group to different audiences. Last week, USA Today's website headlined the revelation that Jessica Lynch had been raped by her Iraqi captors....Finally, yesterday [al-Jazeera] posted a story quoting Iraqi doctors denying the USA Today assertion. Utilizing multiple perspectives via global communications technology allowed me to gain a more in-depth understanding. An open forum with Americans and Iraqis exchanging hopes, dreams and fears may go a long way toward reaching mutually beneficial goals. (Glenn, TCC, Letter 3)

I really enjoyed the comparison that Glenn made between the reporting of the Pvt. Lynch rescue in al-jazeera and USA Today. Not only does it say a great deal about how we communicate but also what we communicate. Technology today is most used to promote what is "fascinating" to the American public, even if it is false. How strange that we have criticized the Iraqi people for printing false information at the whim of a dictator (entered their country to free them of tyranny) and in this land of the free we choose to print false information. Just food for thought. (Erin, Clemson, Letter 3)

While these last two posts indicate the possibility that the accessibility of the Internet and CMC might foster critical thinking, that might be an overly optimistic conclusion. These were after all higher education students trained in critical thinking. However, the information in the Claroline exchange offers a good example of the critical awareness of writing that this diverse group of students arrived at after only a short four-assignment exchange. The form and setup of the exchange created a mixed genre of transformative and transactional writing that appears to have enhanced these students' learning in general as well as increased their awareness of the 
dimensions of writing, CMC, and international expression specifically. In their own words: "we are united in this universal concern” (Fullmer, Clemson, Letter 4) - “the understanding of effective writing skill” (Xing, Chalmers, Letter 3).

\section{More Collaborations}

In Spring 2004, three new groups of students taking three different classes, this time with a focus on literature, engaged in a discussion using the same basic approach and software but with a focus on poetry rather than on communications technology. Although this 2004 Computers and Writing Conference presentation emphasized the Fall 2003 discussion, we made some preliminary remarks about the more recent discussion as well and its implications for collaborations among students from different educational contexts and cultural backgrounds.

Tentative suggestions for further research include the following:

- the discourse features and rhetorical factors in cross-cultural CMC in school-based settings;

- the educational value of computer-mediated, informal, conversational writing;

- the generative and communicative effectiveness of the discussion forum as genre and/or medium in learning contexts;

- the development of writerly knowledge and ability through "writing about writing” and metacognitive analysis in a cross-cultural electronic forum; and closer additional analysis of possible patterns of initial expectations on writing influencing the writer's beliefs and opinion about CMC.

\section{References}

Britton, James; Tony Burgess; Nancy Martin; Alex McLeod; and Harold Rosen. 1975. The Development of Writing Abilities (11-18). London: Macmillan Education.

Britton, James. 1993. Language and Learning. 2nd edition. Portsmouth, New Hampshire: Boynton/Cook.

Bjork, Lennart, Gerd Brauer, Lotte Rienecker, and Peter Stray Jorgensen. Teaching Academic Writing in European Higher Education. Dordrecht, The Netherlands: Kluwer Academic Publishers, 2003.

\section{Acknowledgments}

We would like to thank all our students who participated in this project, each of whom gave us permission to quote from their writing. Special thanks go to Anton Vialle for his gracious troubleshooting and technical expertise in running the Claroline Discussion Board and inviting us to use it. Cross-Cultural Collaborations 